\title{
Die Messen im Tabulaturbuch von Johann Plotz und ihre Verwendung in der Liturgie des evangelischen Gottesdienstes
}

\section{Masses in the Tablature Book of Johann Plotz and their Utilization in the Liturgy of the Evangelical Service}

Marta Hulková / hulkova@phil.uniba.sk

Filozofická fakulta, Univerzita Komenského, Bratislava, SK

\begin{abstract}
According to the specification on the binding of the manuscript, the Tablature Book of Johann Plotz is a "Missal", and the majority of its repertoire, recorded in new German organ tablature notation, corresponds to this designation. Based on its paper, the Tablature Book of Johann Plotz can be dated to the turn of the $16^{\text {th }}$ and the $17^{\text {th }}$ century, and its provenance is probably the geographical region of Silesia-Spiš. The masses recorded here come from the authorial prints of musicians living in the latter half of the $16^{\text {th }}$ century. Side by side, there are masses which originated in a Catholic (Orlando di Lasso, Jacob Handl-Gallus) and in an Evangelical (Johann Knöfel, Leonhard Lechner, Georg Lange, Bartholomäus Gesius) environment. The adoption of Catholic masses into the liturgy of the Evangelical service was a common practice. The mass cycle was shortened to Kyrie and Gloria (Missa brevis), so it could be adjusted to the requirements of the Evangelical service. In its text, the use of the Latin language persisted. In composing masses, the Evangelical musicians followed the liturgical order established by Martin Luther (Deutsche Messe und Ordnung des Gottesdiensts, Wittenberg 1526).
\end{abstract}

\section{Key words}

Tablature Book of Johann Plotz, Silesia, Spiš/Zips/Szepesség, mass, liturgy, Evangelical service 
In der Kirchenmusik des 15. und 16. Jahrhunderts stellt die Messe die wichtigste vokale liturgische Formengattung dar. In ihr wurden die Errungenschaften der kompositorischen Arbeit mehrerer Generationen von Musikern zur Geltung gebracht, die auf dem europäischen Kontinent wirkten. Jeder bedeutende zeitgenössische Komponist war zugleich auch ein Messenkomponist.

Binnen des 16. Jahrhunderts kam es in der christlichen Kirche unter dem Einfluss ihres protestantischen Zweiges zu Modifikationen der Messe, sowohl durch die Reduzierung der Anzahl der Teile des Messordinariums als auch durch die Bevorzugung der Nationalsprachen. In die Entwicklung dieser Formengattung griffen in der zweiten Hälfte des 16. Jahrhunderts auch Anordnungen seitens der katholischen Kirche, konkret des Tridentinischen Konzils ein. ${ }^{1}$

Unter den einzelnen Staatsgebilden der Habsburgermonarchie, in der die deutsche Bevölkerung überwog, gewann die lutherische Reformation im Konfessionsbereich die Oberhand. Die vielfältig verflochtenen politischen und konfessionellen Interessen beeinflussten auch die Musikkultur in Mitteleuropa. Das belegen auch die handschriftlichen Musikalien aus Zips vom Ende des 16. und vom Anfang des 17. Jahrhunderts. ${ }^{2}$ Gleichzeitig ist zu bemerken, dass das sich darin befindende Musikrepertoire bestätigt, der künstlerische Wert der musikalischen Werke bei ihrer Auswahl für die Schreiber (Musiker) entscheidender sei als die konfessionelle Zugehörigkeit des Komponisten. Ein Beweis dafür ist auch die Handschrift, die hier näher vorgestellt wird.

Das Tabulaturbuch von Johann Plotz ist eine Handschrift, die nach der Bezeichnung auf dem Einband ein „Missale“ ist. (Abb. 1) Dem entspricht der größte Teil seines Repertoires, geschrieben in der sog. neuen deutschen Orgeltabulaturnotation. Von der Gesamtzahl der Kompositionen der Handschrift (147) sind über ein Drittel (53) Messen. Das Tabulaturbuch befindet sich in der Bibliothek der Evangelischen Kirche des Augsburger Bekenntnisses (A.B.) auf dem Chor der Kirche in Levoča (dt. Leutschau). ${ }^{3}$ Auf den gemeinsamen Ursprung mit einer weiteren Handschrift dieser Bibliothek, die mit dem Namen Caspar Plotz verbunden ist, ${ }^{4}$ verweisen übereinstimmend zwei Schriftduktus und das verwendete identische Papier. ${ }^{5}$ Der Vergleich des Musikrepertoires dieser Handschriften zeigt, dass sie sich gegenseitig ergänzen. Die Entstehungszeit beider

1 Siehe BLUME, Friedrich. Geschichte der evangelischen Kirchenmusik. Kassel: Bärenreiter-Verlag, ${ }^{2} 1965$; FELLERER, Karl Gustav (Hg.). Geschichte der katholischen Kirchenmusik. Kassel-Basel: Bärenreiter, 2 Bd., ${ }^{3} 1972-$ 1976; FINSCHER, Ludwig - LÜTTEKEN, Laurenz. Die Messe - IV. Mehrstimmige Meßvertonungen bis 1600. In Die Musik in Geschichte und Gegenwart, Ludwig Finscher (hrsg. v.), Bd. 6, Kassel-Stuttgart: Bärenreiter- Metzler 1997, Sp. 184-204.

2 HULKOVÁ, Marta. Levočská zbierka hudobnín [Die Musikaliensammlung von Levoča/Leutschau/Lőcse]. (Diss.), 2 Bd., Bratislava: Filozofická fakulta Univerzity Komenského 1985.

3 Signatur 13990b /2 A/. Im Jahr 2012 wurde das Tabulaturbuch von Johann Plotz restauriert.

4 Tabulaturbuch von Caspar Plotz mit der Signatur 13990a /1 A/.

5 Das Filigran, das eine Jagdtrompete in einem Schild im Tabulaturbuch von Caspar Plotz und im Tabulaturbuch von Johannes Plotz darstellt, stammt laut Viliam Decker aus der Stadt Ravensburg und tritt im Jahr 1595 auf. Siehe DECKER, Viliam. Priesvitky archivu mesta Kremnice v zbierke P. Krisku [Die Wasserzeichen des Archivs der Stadt Kremnitz in der Sammlung von P. Kriska]. Martin: Matica slovenská, 1956, Nr. 70. Vergl. auch HEAWOOD, Edward. Monumenta Chartae Papyraceae I. Watermarks. Hilversum: The Paper Publications Society, 1950, Nr. 2639. 


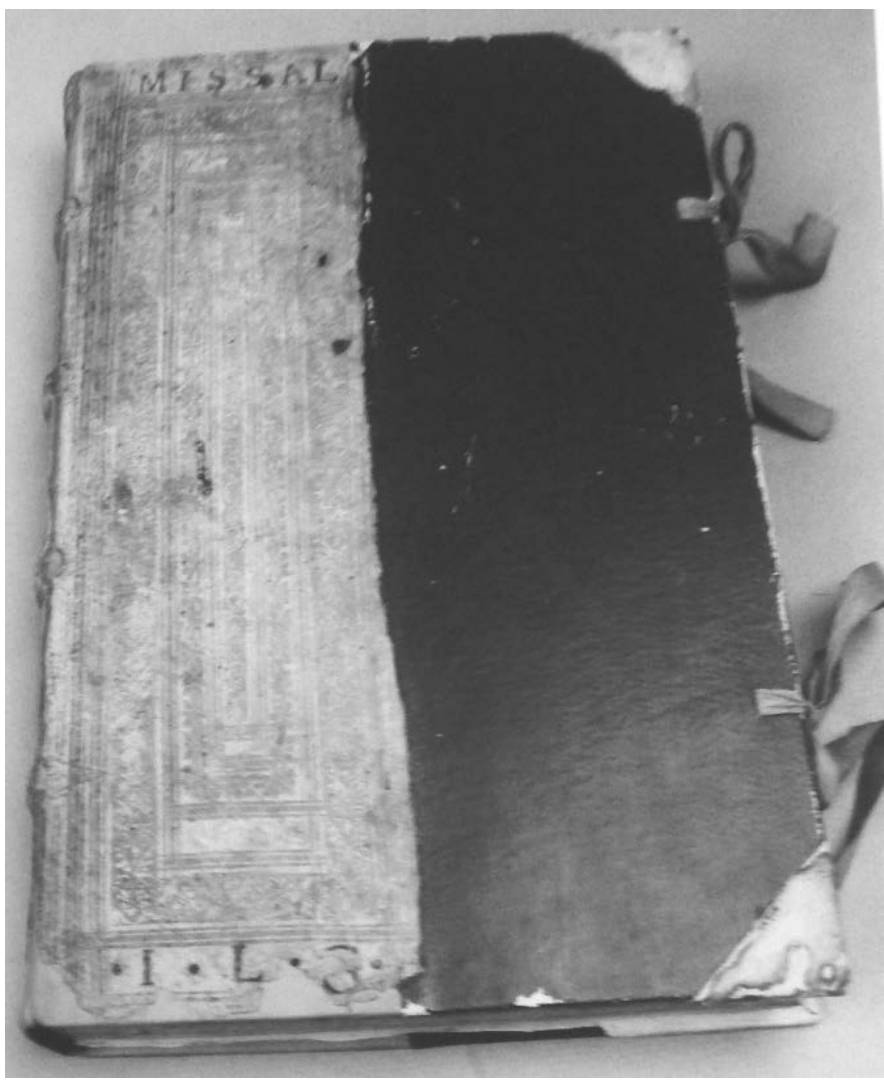

\begin{abstract}
Abb. 1. Tabulaturbuch von Johann Plotz, Sign. 13990b /A 2/ in der Bibliothek der Evangelischen Kirche des Augsburger Bekenntnisse auf dem Chor der Kirche in Levoča.
\end{abstract}

Handschriften (Tabulaturbücher von Caspar und Johann Plotz) legen wir an das Ende des 16. und den Anfang des 17. Jahrhunderts, einerseits nach der Datierung des Papiers, andererseits nach dem erhaltenen Musikrepertoire, das in den meisten Fällen an gedruckte Vorlagen anknüpft. Den Namen dieser beiden Musiker begegnen wir in der musikwissenschaftlichen Literatur erstmals in der Monographie von Friedrich Blume. Er bezeichnete sie als Brüder, ausgehend von den zeitgenössischen Anmerkungen in der Handschrift Sign. Mus. Ms. 40056, die in der Vergangenheit im Besitz der Preußischen Staatsbibliothek in Berlin war und heute in Krakau (Bibliotheka Jagiellońska, Kraków) deponiert ist. ${ }^{6}$ Die nächste Generation deutscher Musikologen Petr Klaus Koch und Wolfram Steude äußerte sich ebenfalls zu Caspar und Johann Plotz und jeweiligen Hypothesen über den Ort des Erwerbs ihrer musikalischen Bildung. ${ }^{7}$ Sie stellen sie in einen

6 „Deutsche Orgeltabulatur von Joh. und Kaspar Plötz aus Brieg“. Siehe BLUME, Friedrich. Die evangelische Kirchenmusik. Potsdam: Akademische Verlagsgesellschaft Athenaion, 1931, S. 129 (Abb. 42), 168, Tafel 5 (Rückseite).

7 Siehe KOCH, Klaus-Peter. Das Claviertabulaturbuch von Caspar und Johannes Plotz. Bemerkungen zu Samuel Scheidt anhand der wieder aufgefundenen Handschrift Mus. Ms. 40056 der ehemaligen Preußischen Staatsbibliothek Berlin. In Beitrage zur musikalischen Quellenforschung. Bad Köstritz: Forschungs- und Gedenkstätte Heinrich-Schütz-Haus,1991, S. 229-243; STEUDE, Wolfram. Bartfelder Handschriften. In Die Musik in Geschichte und Gegenwart, Sachteil 1, Ludwig Finscher (hrsg. v.), Kassel-Stuttgart: Bärenreiter-Metzler 1994, Sp. 1257. 
Zusammenhang mit dem musikalischen Umfeld Samuel Scheidts. Der amerikanische Musikologe Cl. Johnson, der eine weitere Studie über die Handschrift Mus. Ms. 40056 veröffentlichte, ${ }^{8}$ identifiziert sich mit ihren Ansichten. Aufgrund einer Studie der historischen Archivalien in Levoča neigen wir aktuell zu der Ansicht, dass zwischen ihnen größerer Generationsunterschied bestand war, nicht nur ein geschwisterlicher. ${ }^{9}$

Mit den Personen Caspar und Johann Plotz verbinden sich auch die erwähnten, in Levoča erhaltenen Tabulaturbücher. Auf dem Gebiet des slowakischen musikwissenschaftlichen Schrifttums wurden seit den 50er Jahren des 20. Jahrhunderts Studien über diese Handschriften veröffentlicht. Als erster ist Antonín Hořejš und seine Studie „Levočské tabulatúrne sborniky“ [Leutschauer Tabulaturbücher], veröffentlicht 1954, zu erwähnen. ${ }^{10}$ Marta Hulková (1985) und Cleveland Johnson (1989) versuchten, das Musikrepertoire dieser Tabulaturbücher zu identifizieren. In den letzten Jahren entstanden auch Diplomprojekte an der Philosophischen Fakultät der Comenius-Universität in Bratislava, die sich mit den einzelnen Themen beschäftigten. ${ }^{11}$ Eine komplexere Monographie über die Tabulaturbücher von Caspar und Johann Plotz wurde bislang noch nicht veröffentlicht.

Bei der Suche nach Antworten auf die Fragen, d.h. wann, wo, von wem und für wen die Plotzschen Tabulaturbücher angefertigt wurden, bewegen wir uns in den meisten Fällen noch auf Hypothesenniveau. Einer der möglichen Schreiber bzw. Besitzer dieser Tabulaturbücher konnte Caspar Plotz gewesen sein, dessen Name sich auf der ersten Folie des Denkmals 13 990a / 1 A/ zusammen mit der Jahreszahl 1603 befindet. In der Zips sind keine Berichte über das Wirken dieser Musiker erhalten geblieben. Aufgrund der oben erwähnten historischen Notiz in der ursprünglich Berliner Handschrift wissen wir, dass er Organist in der schlesischen Stadt Brieg (heute Brzeg in Polen) war. Eine identische topographische Bemerkung befindet sich dort auch bei dem Namen von Johann Plotz. ${ }^{12}$ Wir nehmen an, dass die in der Berliner Handschrift angeführte Person mit dem Organisten Johann Plotz identisch ist, der in den 40er Jahren des 17. Jahrhunderts in der Zips, in der Stadt Levoča wirkte. ${ }^{13}$ Nach der Schlacht am Weißen Berg wurde

8 JOHNSON, Cleveland. In the Trenches with Johann and Caspar Plotz: a rediscovered Gebrauchstabulatur from the Scheidt circle [online]. DePauw University 2001, Abb. 1. [cit. 2015-9-15]. < http://acad.depauw.edu/ cjohnson/PLOTZ/INDEX.HTML>.

9 Siehe HULKOVÁ, Marta. Die Musikaliensammlung von Bartfeld (Bardejov) und Leutschau (Levoča) Übereinstimmungen und Unterschiede (16. und 17. Jahrhundert). Musicologica Istropolitana, 2003, Jg. 2, S. 83; HULKOVÁ, Marta. Central European Connections of Six Manuscript Organ Tablature Books of the Reformation Era from the Region of Spiš (Zips, Szepes). Studia Musicologica, 2015, Jg. 56, Nr. 1, S. 3-37.

10 HOŘEJŠ, Antonín. Levočské tabulatúrne sborníky. In BURLAS, Ladislav - FIŠER, Ján - HOŘEJŠ, Antonín. Hudba na Slovensku v XVII. storoči. Bratislava: Vydavatelstvo Slovenskej akadémie vied, 1954, S. 99-108.

11 RAJTER, Adrian. Missa Quodlibetica Jacobi Regnarti - príspevok $k$ rekonštrukcii diela [Missa Quodlibetica Jacobi Regnarti - ein Beitrag zur Rekonstruktion des Werkes]. (Dipl. Arbeit), Bratislava: Filozofická fakulta Univerzity Komenského, 1994; FUPŠOVÁ, Marieta. Talianska hudba v Tabulatúrnom zborniku

Caspara Plotza a súvislosti s Promptuarium Musicum Abrahama Schadaea (1611,1612, 1613) [Italienische Musik im Tabulaturbuch von Caspar Plotz und die Zusammenhänge mit Promptuarium Musicum von Abraham Schadaeus (1611,1612, 1613)]. (Dipl. Arbeit), Bratislava: Filozofická fakulta Univerzity Komenského, 2007.

12 JOHNSON, 2001, op. cit.

13 Über den Organisten Johannes Plotz informiert die Hain-Chronik. Siehe Hain Gáspár „Lốcsei krónikája“ / 
Ungarn (einschließlich der Zips) zum Zufluchtsort für evangelische Gebildete, Exilanten aus den Gebieten der Habsburgermonarchie, in denen Gegenreformation gesiegt hatte. Wir nehmen an, dass der Organist von Kežmarok (dt. Käsmark) (seit 1639) Träger des gleichen Nachnamens Georg Plotz, ${ }^{14}$ Johann Plotz die Zips als geeignete Wirkungsstätte empfohlen hatte, der mit ihm in einer verwandtschaftlichen Beziehung gestanden haben konnte. Beide Tabulaturbücher (13990a /1 A/ und 13990b /2 A/) hatte mit größter Wahrscheinlichkeit gerade Johann Plotz aus Schlesien, vielleicht sogar aus der Stadt Brieg (Brzeg), nach Levoča gebracht. Während die Handschrift Sign. 13990a /1 A/ in der Fachliteratur mit dem Namen Caspar Plotz in Verbindung gebracht wird, bezeichnen wir die Handschrift Sign. 13990b /2 A/ mit dem Namen Johann Plotz, seitdem es gelungen ist, den Duktus seiner Schrift zu ermitteln. Er ist in einer gedruckten Einheit der Bibliothek der Evangelischen Kirchengemeinde A.B. in Levoča (Sign. 369) erhalten..$^{15}$ Danach bestimmten wir seinen Schriftduktus im Tabulaturbuch 13990b /2 A/ und damit bestätigte sich gleichzeitig, dass er diese Handschrift besaß. Bislang sind die zwei anonymen Schreiber des Stammrepertoires der Plotzschen Tabulaturbücher nicht bekannt. Wir können nur konstatieren, dass die oben genannte ursprüngliche Berliner Handschrift und die in Levoča erhaltenen Tabulaturdenkmäler keine identischen Schreiber haben.

Die Messen im Tabulaturbuch von Johann Plotz wurden von anonymen Schreibern eingetragen (49) und bilden zusammen mit den eingetragenen Magnificats die ältere Repertoireschicht der Handschrift. Bei der Auswahl des Repertoires wurden Vorlagen von Autorenmusikdrucken aus dem letzten Drittel des 16. und vom Beginn des 17. Jahrhunderts bevorzugt. Aus diesen wählten sie eine größere Anzahl Kompositionen aus, oder schrieben ihren Inhalt komplett ab. Zwischen den Messen und den Magnificats in dem Denkmal befanden sich viele leere Folien, wo Johann Plotz fast den kompletten Inhalt des Musikdruckes Seminarium modulatoriae vocalis von dem deutschen Musiker Otto Gibelius (1645) eintrug. ${ }^{16}$ Die Niederschrift dieser Kompositionen konnte schon in Levoča erfolgt sein, wo Johann Plotz bis 1648 als Organist in der Kirche St. Jakob wirkte. Der beachtliche Zeitabstand zwischen der Zugänglichkeit der Messen und Magnificats

\footnotetext{
Zipserische oder Leutschauerische Chronik von Caspar Hain, (Hg. Jeromos Bal - Jenő Förster - Aurél Kaufmann), Lő́cse 1910-1913, S. 199, 238, 265. Von seinem Wirken in Leutschau zeugen auch die Stadtrechnungen in den Jahren 1641 bis 1648. Siehe Štátny okresný archív - Levoča, Maculatorium pro Anno Domini 1641, Sign. AML XXI A/59, S. 89; AML XXI A/66, S. 93. Diese Angabe gewannen wir aufgrund des Verzeichnisses von NIEDERLAND, Pavol. Záznamy o hudobnom živote v Levoči [Notizen über das Musikleben in Levoča, I]. Levoča 1971, S. 50, 57 (Maschinenschrift).

14 Siehe LIPTÁK, Johann. Geschichte des evangelischen Lyzeums A. B. in Kesmark. Kežmarok: Sauter, 1933, S. 42. Im Rahmen der Anmerkung 48 führt der Autor die Namen der im 17. Jahrhundert in Käsmark wirkenden Organisten an. Hier finden wir den Namen „Georg Plotschius 1639-48“.

15 Siehe HULKOVÁ, 2003, op. cit., Abb. 11, S. 85.

16 Der amerikanische Forscher Cleveland Johnson nimmt an, dass der Autor dieser anonymen dreistimmigen Kompositionen der deutsche Komponist Heinrich Grimm sein konnte. Thomas Synofzik, Autor des thematischen Werkverzeichnis Heinrich Grimm informiert uns unlängst von der wirklichen Vorlage - GIBELIUS, Otto. Seminarium modulatoriae... (1658). Siehe SYNOFZIK, Thomas. Heinrich Grimm (1592/93-1637). Cantilena est loquela canens : Studien zu Überlieferung und Kompositionstechnik mit thematischem Werkverzeichnis. Eisenach: Karl Dieter Wagner, 2000, S. 455.
} 
im Tabulaturbuch von Johann Plotz und dem Erscheinungsjahr der Werke von Gibelius (1645) verweist auch zumindest auf den großen Generationsunterschied zwischen Caspar und Johann Plotz. Also zwischen Caspar, der schon 1603 die Handschrift 13 990a/1 A/ besitzen konnte und Johann, der laut historischer Archivalien 1680 in Levoča starb. ${ }^{17}$

In der Handschrift finden wir Messen von acht Komponisten. Vier davon wirkten in einer katholischen Umgebung: Orlando di Lasso (1532?-1594), Philippe de Monte (1521-1603), Jacob Regnart (1540/45-1599), Jacob Handl-Gallus (1550-1591) und vier in einer evangelischen Umgebung: Johannes Galliculus (1490-1550), Johann Knöfel (1525/30-1617?), Leonhard Lechner (1553-1606), Bartholomäus Gesius (1555/621613).

Das Tabulaturbuch von Johann Plotz beginnt mit einer Messe von Orlando di Lasso. In der Handschrift von ihm finden wir fünf Messen, von denen vier eine gedruckte Form haben. Fünf Parodiemessen auf eine eigene Motette (Missa super Credidi propter /F. 8v-8r/; Missa super Sydus ex claro /F. 10v-13r/; Missa super Veni in hortum meum /F. 232v235r/) erschienen in den Jahren 1574 und $1581^{18}$ und die vierstimmige Missa super Ie ne mange poinct de porcq (F. 0v-2r) im Jahr 1570 im Druck. ${ }^{19}$ Bemerkenswerter hinsichtlich der möglichen Lokalisierung der Entstehung des Tabulaturbuches von Johann Plotz könnte das Vorhandensein der Lassoschen Missa Paschalis (F. 122v-125r) (Abb. 2) sein, die nur in handschriftlicher Form verbreitet wurde. In den zugänglichen Katalogen über historische Musikalien aus Schlesien, konkret aus den Städten Brieg, Lignitz, Breslau, ${ }^{20} \mathrm{bzw}$. vom Territorium der heutigen Slowakei (Bardejov, Levoča, Bratislava, usw.), ${ }^{21}$ kommt diese Messe nicht vor. Bisher ungelöst ist die Frage, wo die Eintragung dieser Komposition in das Tabulaturbuch von Johann Plotz erfolgt sein konnte. Die quellenkritische Edition Missa Paschalis wurde aufgrund einer handschriftlichen Vorlage realisiert, die in der Staats- und Stadtbibliothek Augsburg aufbewahrt ist. ${ }^{22}$ Was die Teile des Messordinariums anbelangt, sind hier zwei Messen von Lasso durch den ersten anonymen Schreiber komplett eingetragen worden. Bei weiteren Messen notiert der zweite anonyme Schreiber eine verkürzte Version: Kyrie und Gloria, (bzw. Kyrie, Gloria, Sanctus, Agnus). Dies

17 MATÚŠ, František (ed.). Tabulatúrny zbornik Samuela Marckfelnera / Tabulaturbuch des Samuel Marckfelner (Výber / Auswahl). Bratislava: OPUS, 1981, S. 5.

18 LASSO, Orlando di. Patrocinium musices ... missae aliquot quinque vocum... Monachii: Adamus Berg, 1574 (RISM A/I/5 L 873); ders. Liber missarum 4, 5 vocum... Noribergae: Imprimebatur typis Gerlachianis, 1581 (RISM A/I/5 L 924).

19 LASSO, Orlando di. Quinque missae suavissimis ... Venetia: Claudio Correggio, 1570 (RISM A/I/5 L 831).

20 KUHN, Friedrich. Beschreibendes Verzeichnis der alten Musikalien - Handschriften und Druckwerke des Gymnasiums zu Brieg. Leipzig: Breitkopf \& Härtel, 1897; PFUDEL, Ernst. Die Musik-Handschriften der Königl. RitterAkademie zu Liegnitz. In Beilage zu den Monatsheften für Musikgeschichte, Robert Eitner (ed.), 1. Bd., Leipzig: Breitkopf et Haertel, 1886, S. 5-74; BOHN, Emil. Die musikalischen Handschriften des XVI. und XVII. Jahrhunderts in der Stadtbibliothek zu Breslau. Breslau: Commissions-Verlag von Julius Hainauer, 1890.

21 MURÁNYI. Róbert Árpád. Thematisches Verzeichnis der Musiksammlung von Bartfeld (Bártfa). Bonn: Gudrun Schröder Verlag, 1991; HULKOVÁ, 1985, op. cit.; KALINAYOVÁ, Jana und Autorenkollektiv. Musikinventare und das Repertoire der mehrstimmigen Musik in der Slowakei im 16. und 17. Jahrhundert. Bratislava: SNM-Hudobné múzeum, 1995.

22 Sign. Tonk.Schl.17, siehe auch LASSO, Orlando di. Missa Paschalis, 5vv, (MS 1576), Kurt von Fischer (ed.), Sämtliche Werke, neue Reihe, Kassel: Bärenreiter, 1968, Jg IX, Bd. 7, Nr. 48, S. 131. 

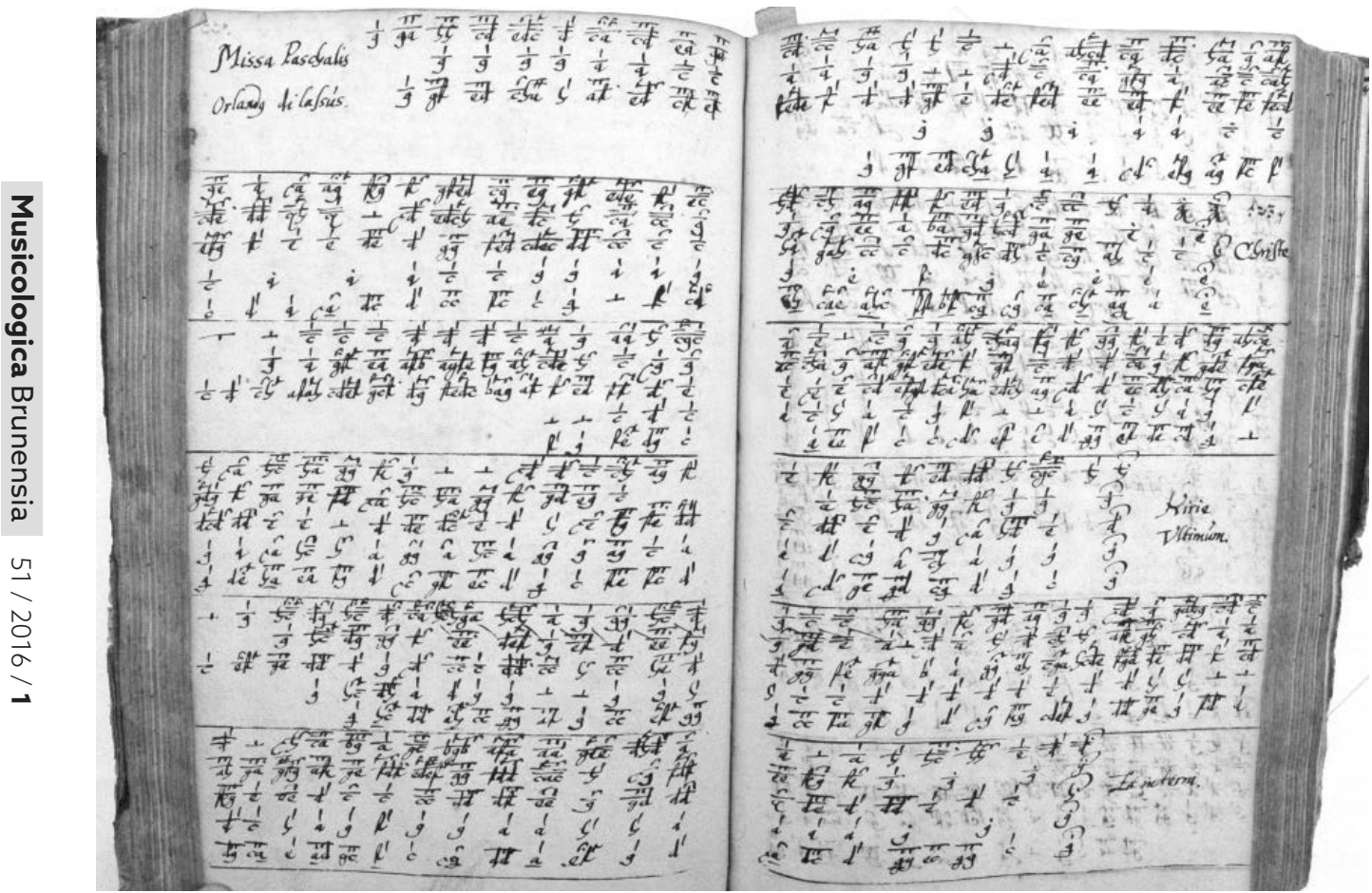

Abb. 2. Missa Paschalis von Orlando di Lasso, Tabulaturbuch von Johann Plotz (Sign. 13990b /A 2/, F. 122v-125r) in der Bibliothek der Evangelischen Kirche des Augsburger Bekenntnisse auf dem Chor der Kirche in Levoča.

belegt, dass er in den Intentionen der Liturgie des evangelischen Gottesdienstes dachte. Die Lassos Schaffen war auf dem Territorium Oberungarns, also auf dem Gebiet der heutigen Slowakei allgemein verbreitet, sowohl im katholischen als auch im evangelischen Umfeld und wurde auch in der ersten Hälfte des 17. Jahrhunderts gepflegt. ${ }^{23}$

Von Philippe de Monte (1521-1603), einem flämischen Musiker im Dienste der habsburgischen Höfe in Wien (bei Maximilan II.) und in Prag (bei Rudolph II.) befindet sich im Tabulaturbuch von Johann Plotz die Missa super Nasce la pena mia (F. 241v-245r). Sie ist mit allen Teilen des Messordinariums eingetragen. In Mitteleuropa sind sogar sechs Eintragungen dieser Parodiemesse in handschriftlicher Form erhalten - Berlin, Brno, Brussel, Gdańsk, Wien, Wrocław. ${ }^{24}$ Ihnen können weitere vier Erscheinungen auf dem

23 MURÁNYI, op. cit.; HULKOVÁ, 1985, op. cit.; KALINAYOVÁ und Autorenkollektiv, op. cit.

24 Siehe LINDELL, Robert. - MANN, Brian R. Monte, Philippe de. In The New Grove Dictionary of Music and Musicians, Stanley Sadie (ed.), London: Oxford University Press, ${ }^{2} 2001$, zv. 17, S. 20; POPIGINIS, Danuta - SZLAGOWSKA, Danut. Musicalia Gedanensis. Rekopisy muzyczne z XVI i XVII wieku w zbiorach Biblioteki Gdańskiej. Gdańsk: Polskiej Akademii Nauk, 1990, S. 213. Siehe die Quellenedition: MONTE, Philippi de: Missa Nasce la pena mia. Carolus van den Borren (ed.), Düsseldorf: Sumptibus L. Schwann, (=Opera Omnia 10), reprint New York: Broude Brothers, 1965. 


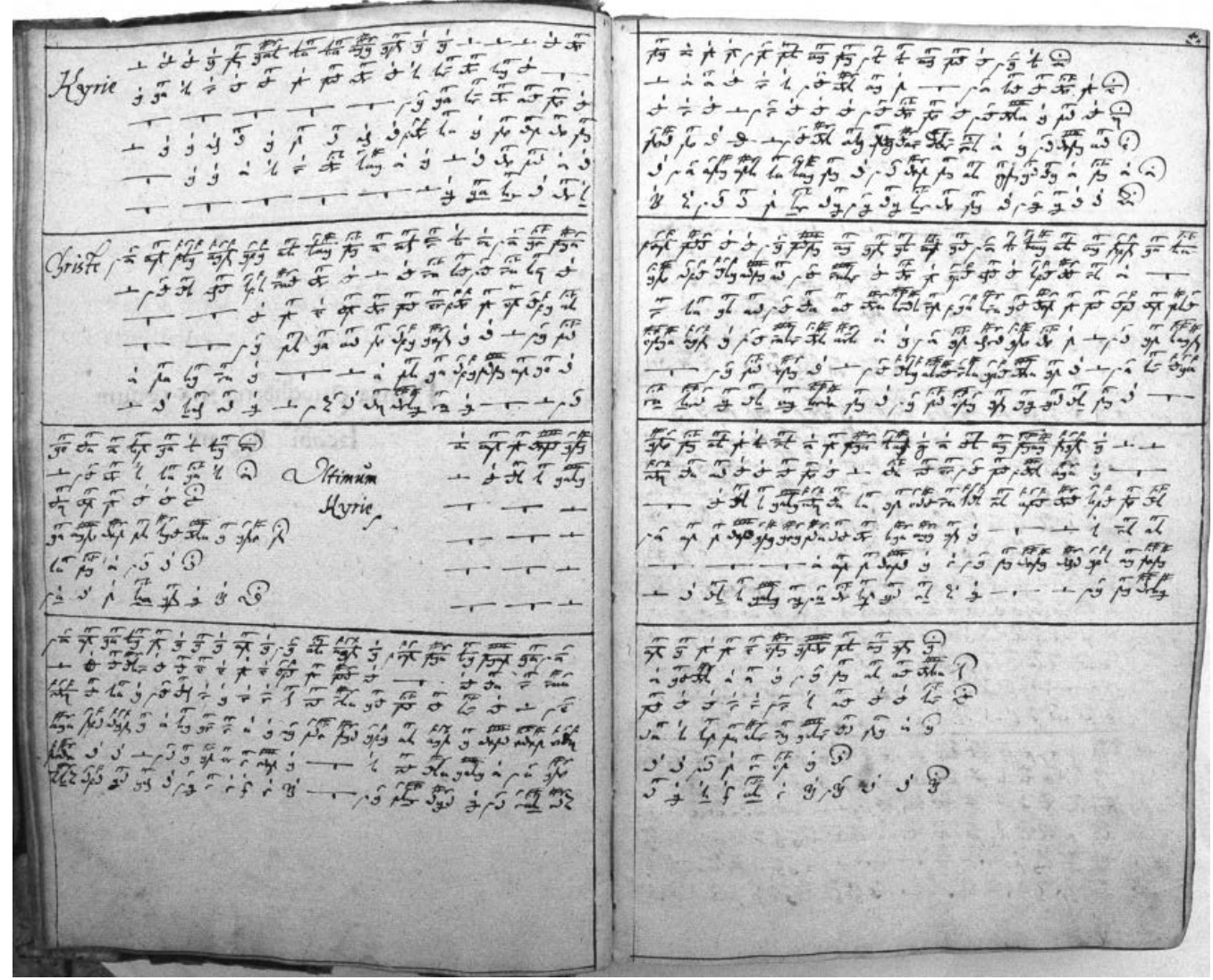

Abb. 3. Missa Quodlibetica von Jacob Regnart, Tabulaturbuch von Johann Plotz (Sign. 13990b /A 2/, F. 4v-5r) in der Bibliothek der Evangelischen Kirche des Augsburger Bekenntnisse auf dem Chor der Kirche in Levoča.

Gebiet heutiger Slowakei hinzugefügt werden. Außer dem Tabulaturbuch von Johann Plotz auch im Kaschauer polyphonen Sammelband III, in Stimmheften der Bartfelder Musikaliensammlung und bei den Preßburger Franziskanern. ${ }^{25}$ Montes sechsstimmige Parodiemesse Nasce la pena mia wurde durch das Madrigal des italienischen Musikers Alessandro Striggia inspiriert und in einem reifen franko-flämischen polyphonen Stil komponiert.

Von Jacob Regnart (1540/45-1599), einem weiteren bedeutenden Musiker, der am Hofe Rudolph II. in Prag wirkte, finden wir im Tabulaturbuch von Johann Plotz zwei Messen. Als anonyme Komposition zweimal eingetragen (F. 127v; F. 155v) ist die Missa super Exultate, die in dem Regnartschen Musikdruck Missae sacrae ad imitationem... identifiziert

25 Im Tabulaturbuch von Johann Plotz, F. 241v-245r (Bibliothek der Evangelischen Kirche des A. B., Levoča, Sign. 13990b /2 A/); in Košický polyfónny zbornik III [Kaschauer polyphonen Sammelband III], F. 70v-86r (Východoslovenské múzeum, Košice, Sign. H 67.054); in Ms. mus. Bártfa 15, Nr. 4 (Országos Széchényi könyvtár, Budapest); Konvolut aus der Bibliothek des Franziskanerkloster in Pressburg, Ms. Teil, ohne Pagination (Slovenská národná knižnica, Martin, Sign. Bap 26860 /olim 4717/). 
werden konnte (1602). ${ }^{26}$ Das erste Mal sind auf der F. 127v aus dem Messordinarium die Teile Kyrie und Gloria eingetragen und bei der zweiten Eintragung auf F. 155v nur das Kyrie. Regnarts Missae sacrae ad imitationem besaßen während des 17. Jahrhunderts nur Preßburger evangelische Gläubige. ${ }^{27}$ Bemerkenswerter ist das Vorkommen einer weiteren, nur in handschriftlicher Form erfassten Messe von J. Regnart, die Missa Quodlibetica (Abb. 3), die mit allen Teilen des Messordinariums aufgezeichnet ist. Walter Pass war während der Aufstellung des Thematischen Katalogs der Werke Regnarts nur die Altstimme dieser Messe bekannt, die in der Musikabteilung der Nationalmuseums (České muzeum hudby $)^{28}$ in Prag aufbewahrt wird, bekannt. Ein weiteres mögliches Exemplar in der Stadt Wrocław bezeichnete er als nicht zugänglich. ${ }^{29}$

Von dem slowenischen Musiker Jacob Handl-Gallus (1550-1591), der in Mähren, und an seinem Lebensabend auch in Prag wirkte, sind im Tabulaturbuch von Johann Plotz fünfzehn Messen eingetragen (F. 56v-110r). Der erste anonyme Schreiber trug außer der letzten (Missa canonica) alle anderen Messen aus seinen Selectiones quedam missae ein, die 1580 in vier Bänden in Prag bei Georg Nigrini herausgegeben wurden. ${ }^{30}$ Es handelt sich um Parodiemessen, einerseits auf eigene Motetten, aber häufiger nach einem übernommenen Modell, z.B. von Philipp Verdelot, Clemens non Papa, O. di Lasso, usw. Von den Teilen des Messordinariums ließ der Schreiber nur das Credo weg, worauf besonders auch Marko Motnik in seinem Thematischen Katalog von J. Handl-Gallus verwies. ${ }^{31}$

Johannes Galliculus (1490-1550?) ist mit einer Messe (F. 236v-241r) vertreten. Er ist der älteste von allen Musikern, deren Werke im Tabulaturbuch von Johannes Plotz zu finden sind. Er gehört zu den ersten lutherischen Musikern und trug wesentlich zur Bildung der lateinischen mehrstimmigen frühlutherischen Ordinarien- und Proprienkompositionen bei. Seine vierstimmige Messe, die 1539 in Wittenberg bei Georg Rhaw in dem Sammeldruck Officia Paschalia, De resurrectione et Ascensione Domini erschien, ${ }^{32}$ hatte der anonyme Schreiber zwischen die Messen O. di Lasso und Ph. de Monte geordnet.

Die anonym aufgezeichneten 7 lateinischen Messen von Johann Knöfel (1525/30-1617?) identifizierte Cleveland Johnson im Tabulaturbuch von Johann Plotz (F. 24v-54r). ${ }^{33}$ Sie waren von dem Druck Cantus choralis abgeschrieben, erschienen 1575 in Nürnberg, den Knöfel dem Stadtrat in Breslau (heute Wrocław) gewidmet hatte. Knöfel gehörte zu den bedeutenden schlesischen lutherischen Musikern, der am Ende seines aktiven Lebens

26 REGNART, Jacob. Missae sacrae ad imitationem... Frankfurt: Nikolaus Stein, 1602 (RISM A/I/7 R 734/9). Siehe auch PASS, Walter. Thematischer Katalog sämtlicher Werke Jacob Regnarts (ca. 1540-1599). (=Tabulae Musicae Austriacae 5), Wien, Köln, Graz: Hermann Böhlau in Kommission, 1969, Nr. 31.

27 KALINAYOVÁ und Autorenkollektiv, op. cit., S. 65.

28 Sign. AZ 36 (IV.F.1. Anhang). Aktuelle Benennung der Institution: Národni muzeum-České muzeum hudby.

29 Sign. Ms. 99A, Nr. 11 (nach Emil Bohn). PASS, op. cit., Nr. 27.

30 HANDL-GALLUS, Jacob. Selectiones quedam missae... 4 Bd., Prag: Georg Nigrini, 1580 (RISM A/I/4 H $1976,1977,1978,1979)$

31 Siehe MOTNIK, Marko. Jacob Handl-Gallus. Werk - Überlieferung - Rezeption mit thematischem Katalog. Tutzing: Hans Schneider, 2012 , Katalog 1-15, S. 463-470.

32 Siehe Officia Paschalia, De resurrectione... Wittenberg: Rhaw, 1539 (RISM B/I/1 1539 ${ }^{14}$ ), f. a3v.

33 KNÖFEL, Johann. Cantus Choralis. Musicis Numeris... Noribergae: Dietrich Gerlach, 1575 (RISM A/I/5 K 990). Siehe JOHNSON, 1989, S. 68. 
in Prag wirkte (seit 1592). Seine fünfstimmigen Messen, die für die Hauptfeste des Kirchenjahres komponierte und Teile des Ordinariums und Propriums enthaltend, gelten als erfolgreiche Bewältigung der kompletten Struktur des lutherischen Gottesdienstes. ${ }^{34}$

Von Leonhard Lechner (1553-1606) befinden sich im Tabulaturbuch von Johann Plotz zwei Parodiemessen (Missa super Domine Domunis noster, Missa super Non fu mai cervo), die eine Abschrift aus seinem Musikdruck Liber missarum sex et quinque vocum... aus dem Jahr 1584 sind.$^{35}$ Lechner wuchs musikalisch am Münchener Hof auf. Als 18-jähriger trat er zum lutherischen Glauben über und wirkte dann in Nürnberg und in Stuttgart in einem evangelischen Umfeld. ${ }^{36}$ Als Modelle für die Parodiemessen dienten ihm die Motette Domine Dominus noster von O. di Lasso und das Madrigal von Luca Marenzio Non fu mai cervos.

16 Messen für fünf-, sechs- und achtstimmige Besetzung wählte der zweite anonyme Schreiber des Tabulaturbuches von Johann Plotz von dem Musikdruck Opus plane novum cantionum ecclesiasticarum $^{37}$ von Bartholomäus Gesius (1551/62-1613) aus. In den meisten Fällen bei dem lutherischen Musiker B. Gesius haben wir nur mit der missa brevis (Kyrie, Gloria) zu tun.. Ähnlich wie die vorherigen Musiker komponierte er ebenfalls Parodiemessen. Als Vorlage wählte er Kompositionen etwa von Orlando di Lasso, Luca Marenzio, Georg Lange. Das Gesiussche Schaffen aus der Zeit seines Wirkens in der Marienkirche in Frankfurt an der Oder war sowohl in Schlesien als auch in der Zips sehr beliebt und verbreitet. ${ }^{38}$

Wir nehmen an, dass die ältere Repertoireschicht des Tabulaturbuches von Johann Plotz (zu der auch die Messen gehören) höchstwahrscheinlich in Schlesien entstanden ist. Dafür spricht zum einen die dortige Zugänglichkeit der meisten historischen Drucke, die die zwei anonymen Schreiber des Tabulaturbuches zu ihrer Verfügung haben mussten, sowie historische Angaben, die Caspar und Johann Plotz mit der Stadt Brieg in Verbindung bringen. Bisher haben wir die Situation im Gebiet Zips-Scharosch (slow. SpišŠariš), konkret in den fünf königlichen Städten - Levoča, Bardejov, Prešov, Sabinov und Košice (dt. Leutschau, Bartfeld, Eperies, Zeben, Kaschau) - erfasst, deren konfessionelle Zugehörigkeit zum Protestantismus im Jahr 1549 in dem Dokument Confessio Pentapolitana (1549) verankert war. ${ }^{39}$ Levoča, in dem das Tabulaturbuch von Johann Plotz erhalten geblieben ist, betrachten wir auch als einen Ort, in dem die oben erwähnten Messen während der evangelischen Gottesdienste in der ersten Hälfte des 17. Jahrhunderts auf-

34 MÖLLER, Hartmut. Knöfel, Johann. In Die Musik in Geschichte und Gegenwart. Personenteil 10. Ludwig Finscher (hrsg. v.), Kassel-Stuttgart: Bärenreiter-Metzler 2003, Sp. 347-349.

35 LECHNER, Leonhard. Liber missarum sex et quinque vocum... Noribergae: Dietrich Gerlach, 1584 (RISM A/I/5 L 1298)

36 AMELN, Konrad. Leonhard Lechner. In Die Musik in Geschichte und Gegenwart. Personenteil 10. Ludwig Finscher (hrsg. v.), Kassel-Stuttgart: Bärenreiter-Metzler 2003, Sp. 1409-1414.

37 GESIUS, Bartholomäus. Opus plane novum cantionum ecclesiasticarum... Frankfurt/Oder: Friedrich Hartmann, 1613 (RISM A/I/3 G 1707)

38 Siehe BOHN, op. cit.; HULKOVÁ, 1985, op. cit.; MURÁNYI, op. cit.

39 HAJDUK, Andrej. Die Confessio Pentapolitana. Lutherische Kirche in der Welt, 1982, Jg. 29, S. 139-149. Siehe auch HULKOVÁ, Marta. Hudobné tlače s viachlasnou hudbou zo 16. a 17. storočia v Pentapolitane [Die Musikdrucke mit mehrstimmiger Musik aus dem 16.-17. Jh. in Pentapolitana]. In Kniha 2008 - Zbornik o problémoch a dejinách knižnej kultúry, Miroslava Domová (ed.), Martin: Slovenská národná knižnica, 2008, S. 79-96. 
geführt werden konnten. Die dortigen Stadtschulen waren zeitgemäß auf einem hohen Niveau auch hinsichtlich des Unterrichts im Fach Musik. ${ }^{40}$

Es ist eine anspruchsvolle Aufgabe, die historischen liturgischen Gewohnheiten während des evangelischen Gottesdienstes zu rekonstruieren. Luthers Absicht, die katholische Messe zu modifizieren, war in seinen Schriften Formula Missae et Communionis (1523) und Deutsche Messe (1526) verankert. ${ }^{41} \mathrm{Zu}$ den geforderten Veränderungen in der Liturgie des evangelischen Gottesdienstes kam es schrittweise, üblich waren mehrere örtliche Varianten. Eine wichtige Rolle bei der Vereinheitlichung der Liturgie des evangelischen Gottesdienstes spielte die Kirchenordnung (Agenden). Aus dem 16. Jahrhundert erfassen wir in den Zipser historischen Bibliotheken mehrere deutsche Kirchenordnungen aus Wittenberg, Nürnberg, Jena. (Abb. 4) Z.B. in der Bibliothek der evangelischen Kirche A.B. in Levoča ist die allgemein verbreitete Kirchenordnung von Veit Dietrich, ${ }^{42}$ einem Nürnberger Prediger erhalten, der in einem engen Kontakt zu

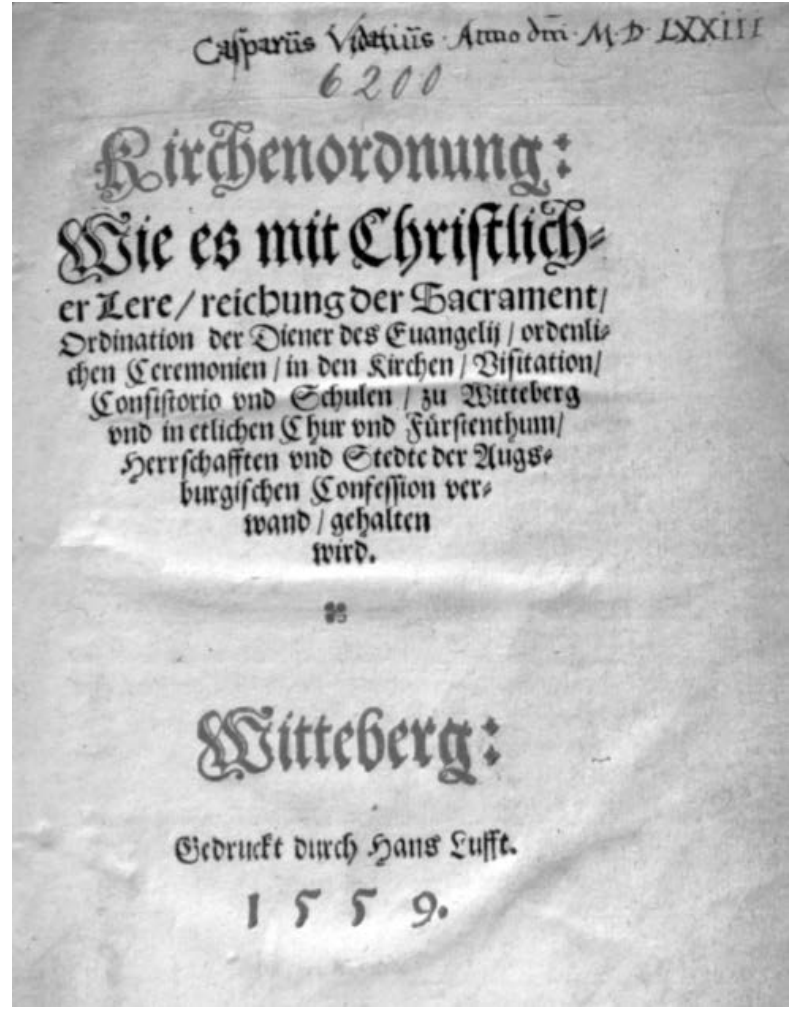

Abb. 4. Agenda. Das ist Kirchenordnung... Jehna: Salamon Richtzenhan, 1600. In der Bibliothek der Evangelischen Kirche des Augsburger Bekenntnisse auf dem Chor der Kirche in Levoča, Sign. 6005.

40 HULKOVÁ, Marta. Beitrag zur Problematik der Musikerziehung in den Stadtschulen auf dem Gebiet der Slowakei im 16. Jahrhundert. Musicologica Istropolitana, 2005, Jg. 4, S. 41-59.

41 Siehe BLUME, 1931, op. cit., S. 27-31.

42 Sign. 7378 in der Bibliothek der Evangelischen Kirche des Augsburger Bekenntnisse auf dem Chor der Kirche in Levoča: VEIT, Dietrich. Agend Büchlein... Nürnberg: Berg \& Newber, 1553; weitere Sign. 6005: Agenda. Das ist Kirchenordnung... Jehna: Salamon Richtzenhan, 1600. 
Martin Luther und Philipp Melanchton stand.

Das oben genannte Dokument Confessio Pentapolitana, das auf Wunsch des Herrschers Ferdinand I. zur Verteidigung des Augsburger Bekenntnisses erarbeitet wurde, distanziert sich nicht von der katholischen Kirche, es wird vielmehr als eine ihrer möglichen progressiven Richtungen präsentiert. Wenn wir von den historischen Kirchenordnungen der Regionen Zips und Scharosch ausgehen, z.B. von dem Beschluss der Synode zu Prešov (dt. Eperies) Canones synodi Eperiensis de doctrina et ritibus aus dem Jahr 1546, von der Confessio Scepusiana aus dem Jahr 1569, oder der sog. Leutschauer Konvention, die aus dem 17. Jahrhundert stammt, zeigt sich in ihnen das Bestreben, die Konflikte mit der katholischen Kirchenobrigkeit zu überbrücken. Einerseits wurde an eingebürgerte liturgische Gewohnheiten angeknüpft und andererseits passte man sich auch an die Wittenberger Kirchenordnung an. Das bemerkenswerte Dokument des Stadtmagistrats von Košice (dt. Kaschau) aus dem Jahr 1559 veröffentlichte Vojtech Wick mit konkreten Hinweisen für den neugewählten ungarischen evangelischen Pfarrer, nach dem die Liturgie aus folgenden Teilen bestand: Introitus - Kyrie - Gloria - Epistel (auf Ungarisch) - das Evangelium (auf Ungarisch) - Hymnus prosa (Altus auctor omnium) - Bitten, Collecta (auf Ungarisch). ${ }^{43}$

Der erste anonyme Schreiber des Tabulaturbuchs von Johann Plotz trug alle Teile des Messordinariums, stellenweise auch des Propriums ein. Das deutet daran, dass die Handschrift ursprünglich in einem solchen Kirchenumfeld gedient haben könnte, in dem die Tradition der Liturgie der katholischen Messe noch stärker weiterlebte. Der zweite anonyme Schreiber, der an den leeren Stellen nach dem ersten noch weitere Kompositionen eintrug und dann ab der Folie 122v kontinuierlich mit der Eintragung fortfuhr, notierte nur eine verkürzte Form des Messordinariums (Kyrie, Gloria). Am Beispiel des Tabulaturbuchs von Johann Plotz konnten wir beobachten, dass die Musikhandschriften die regionalen Spezifika eindeutiger nachweisen können, als die erhaltenen Musikdrukke. Die Repertoireauswahl bei den Handschriften verrät nicht nur die Erfahrenheit der Schreiber, der Kantoren und Organisten damaliger Zeit, aber auch ortsgebundene Gewohnheiten, die sich mit der Zeit wandeln und durch Migration der Musiker auch stärker modifizieren konnten. ${ }^{44}$

\section{Bibliography}

\section{Sources}

Hudobný konvolút z bratislavskej františkánskej knižnice [Musik-Konvolut aus der Bibliothek des Franziskanerklosters in Pressburg], Ms. Theil, ohne Pagination - Slovenská národná knižnica, Martin, Sign. Bap 26860 /olim 4717/.

43 Nach der Übersetzung von der lateinischen Sprache siehe WICK, Vojtech. Dóm svätej Alžbety v Košiciach [Dom der Heiligen Elisabeth in Košice]. Košice: Nákladom mesta Košíc, 1936, S. 65-70.

44 VEGA Projekt Nr. 1/0699/2016-19. 
Košický polyfónny zbornik III [Kaschauer polyphonen Sammelband III], (Ms.) - Východoslovenské múzeum, Košice, Sign. H 67.054

Maculatorium pro Anno Domini 1641 - Štátny okresný archív, Levoča, Sign. AML XXI A/59; AML XXI A/66.

Ms. mus. Bártfa 15 - Országos Széchényi Könyvtár [National Széchényi Bibliothek], Budapest.

Tabulatúrny zbornik Caspara Plotza [Tabulaturbuch von Caspar Plotz], (Ms.) - Knižnica evanjelickej cirkvi a.v., Levoča, Sign. 13990a / 1 A/.

Tabulatúrny zbornik Johanna Plotza [Tabulaturbuch von Johann Plotz], (Ms.) - Knižnica evanjelickej cirkvi a.v., Levoča, Sign. 13990b /2 A/.

\section{Literature}

AGENDA. Das ist Kirchenordnung... Jehna: Salamon Richtzenhan, 1600.

AMELN, Konrad. Leonhard Lechner. In Die Musik in Geschichte und Gegenwart. Personenteil 10. Ludwig Finscher (hrsg. v.), Kassel-Stuttgart: Bärenreiter-Metzler, 2003, Sp. 1409-1414.

BLUME, Friedrich. Die evangelische Kirchenmusik. Potsdam: Akademische Verlagsgesellschaft Athenaion, 1931.

BLUME, Friedrich. Geschichte der evangelischen Kirchenmusik. Hg. unter Mitarbeit von. Ludwig Finscher [u.a.]. Kassel: Bärenreiter-Verlag, ${ }^{2} 1965$.

BOHN, Emil. Die musikalischen Handschriften des XVI. und XVII. Jahrhunderts in der Stadtbibliothek zu Breslau. Breslau: Commissions-Verlag von Julius Hainauer, 1890.

DECKER, Viliam. Priesvitky archívu mesta Kremnice v zbierke P. Križku [Die Wasserzeichen des Archivs der Stadt Kremnitz in der Sammlung von P. Križku]. Martin: Matica slovenská, 1956.

FELLERER, Karl Gustav (Hg.). Geschichte der katholischen Kirchenmusik. Kassel-Basel: Bärenreiter, 2 Bd., ${ }^{3} 1972-1976$.

FINSCHER, Ludwig. - LÜTTEKEN, Laurenz. Die Messe - IV. Mehrstimmige Messvertonungen bis 1600. In Die Musik in Geschichte und Gegenwart, Ludwig Finscher (hrsg. v.), Kassel-Stuttgart: Bärenreiter-Metzler 1997, Bd. 6, Sp. 184-204.

FUPŠOVÁ, Marieta. Talianska hudba v Tabulatúrnom zborniku Caspara Plotza a súvislosti s Promptuarium Musicum Abrahama Schadaea (1611, 1612, 1613) [Italienische Musik im Tabulaturbuch von Caspar Plotz und die Zusammenhänge mit Promptuarium Musicum von Abraham Schadaeus (1611, 1612, 1613)]. (Dipl. Arbeit), Bratislava: Filozofická fakulta Univerzity Komenského, 2007.

GESIUS, Bartholomäus. Opus plane novum cantionum ecclesiasticarum... Frankfurt/Oder: Friedrich Hartmann, 1613. (RISM A/I/3 G 1707)

HAIN GÁSPÁR „Löcsei krónikája“ / Zipserische oder Leutschauerische Chronik von Caspar Hain. (Hg. Jeromos Bal - Jenő Förster - Aurél Kaufmann), Lőcse: Szepesmenyei Töténelmi Társulat, 1910-1913.

HAJDUK, Andrej. Die Confessio Pentapolitana. Lutherische Kirche in der Welt, 1982, Jg. 29, S. 139149.

HANDL-GALLUS, Jacob. Selectiones quedam missae... 4 Bd., Prag: Georg Nigrini, 1580. (RISM A/I/4 H 1976, 1977, 1978, 1979)

HEAWOOD, Edward. Monumenta Chartae Papyraceae I. Watermarks. Hilversum: The Paper Publications Society, 1950.

HOŘEJŠ, Antonín. Levočské tabulatúrne sborníky. In BURLAS, Ladislav - FIŠER, Ján - HOŘEJŠ, Antonín. Hudba na Slovensku v XVII. storoči. Bratislava: Vydavatel'stvo Slovenskej akadémie vied, 1954, s. 99-108. 
HULKOVÁ, Marta. Levočská zbierka hudobnín [Die Musikaliensammlung von Levoča/Leutschau/ Lőcse]. (Diss.), 2 Bd., Bratislava: Filozofická fakulta Univerzity Komenského, 1985.

HULKOVÁ, Marta. Die Musikaliensammlung von Bartfeld (Bardejov) und Leutschau (Levoča) Übereinstimmungen und Unterschiede (16. und 17. Jahrhundert). Musicologica Istropolitana, 2003, Jg. 2, S. 51-113.

HULKOVÁ, Marta. Beitrag zur Problematik der Musikerziehung in den Stadtschulen auf dem Gebiet der Slowakei im 16. Jahrhundert. Musicologica Istropolitana, 2005, Jg. 4, S. 41-59.

HULKOVÁ, Marta. Central European Connections of Six Manuscript Organ Tablature Books of the Reformation Era from the Region of Spiš (Zips, Szepes). Studia Musicologica, 2015, Jg. 56, Nr. 1, S. 3-37.

JOHNSON, Cleveland. Vocal Compositions in German Organ Tablatures 1550-1650. A Catalogue and Commentary. (=Outstanding Dissertations in Music from British Universities), New York et London: Garland Publishing, 1989.

JOHNSON, Cleveland. In the Trenches with Johann and Caspar Plotz: a rediscovered Gebrauchstabulatur from the Scheidt circle [online]. DePauw University, 2001. [cit. 2015-9-15]. < http://acad.depauw. edu/ ${ }^{\sim}$ cjohnson/PLOTZ/INDEX.HTML>.

KALINAYOVÁ, Jana und Autorenkollektiv. Musikinventare und das Repertoire der mehrstimmigen Musik in der Slowakei im 16. und 17. Jahrhundert. Bratislava: SNM-Hudobné múzeum, 1995.

KNÖFEL, Johann. Cantus Choralis. Musicis Numeris... Noribergae: Dietrich Gerlach, 1575. (RISM A/I/5 K 990)

KOCH, Klaus-Peter. Das Claviertabulaturbuch von Caspar und Johannes Plotz. Bemerkungen zu Samuel Scheidt anhand der wieder aufgefundenen Handschrift Mus. Ms. 40056 der ehemaligen Preußischen Staatsbibliothek Berlin. In Beitrage zur musikalischen Quellenforschung. Bad Köstritz: Forschungs- und Gedenkstätte Heinrich-Schütz-Haus,1991, S. 229-243.

KUHN, Friedrich. Beschreibendes Verzeichnis der alten Musikalien - Handschriften und Druckwerke des Gymnasiums zu Brieg. Leipzig: Breitkopf \& Härtel, 1897.

LASSO, Orlando di. Patrocinium musices ... missae aliquot quinque vocum... Monachii: Adamus Berg, 1574. (RISM A/I/5 L 873)

LASSO, Orlando di. Liber missarum 4, 5 vocum... Noribergae: Imprimebatur typis Gerlachianis, 1581. (RISM A/I/5 L 924)

LASSO, Orlando di. Quinque missae suavissimis... Venetia: Claudio Correggio, 1570. (RISM A/I/5 L 831)

LASSO, Orlando di. Missa Paschalis, 5vv, (MS 1576), Kurt von Fischer (ed.). In Sämtliche Werke, neue Reihe, Kassel: Bärenreiter, 1968, Jg IX, Bd. 7, Nr. 48, S. 131.

LECHNER, Leonhard. Liber missarum sex et quinque vocum... Noribergae: Dietrich Gerlach, 1584. (RISM A/I/5 L 1298)

LINDELL, Robert. - MANN, Brian R. Monte, Philippe de. In The New Grove Dictionary of Music and Musicians, Stanley Sadie (ed.), London: Oxford University Press, ${ }^{2} 2001$, Bd. 17, S. 20.

LIPTÁK, Johann. Geschichte des evangelischen Lyzeums A. B. in Kesmark. Kežmarok: Sauter, 1933.

MONTE, Philippi de: Missa Nasce la pena mia. Carolus van den Borren (ed.), Düsseldorf: Sumptibus L. Schwann, (=Opera Omnia 10), reprint New York: Broude Brothers, 1965.

MOTNIK, Marko. Jacob Handl-Gallus. Werk - Überlieferung - Rezeption mit thematischem Katalog. Tutzing: Hans Schneider, 2012.

MÖLLER, Hartmut. Knöfel, Johann. In Die Musik in Geschichte und Gegenwart. Personenteil 10. Ludwig Finscher (hrsg. v.), Kassel-Stuttgart: Bärenreiter-Metzler, 2003, Sp. 347-349. 
MURÁNYI. Róbert Árpád. Thematisches Verzeichnis der Musiksammlung von Bartfeld (Bártfa). Bonn: Gudrun Schröder Verlag, 1991.

NIEDERLAND, Pavol. Záznamy o hudobnom živote v Levoči [Notizen über das Musikleben in Levoča, I]. Levoča 1971, (Maschinenschrift).

OFFICIA PASCHALIA, De resurrectione... Wittenberg: Rhaw, 1539. (RISM B/I/1 153914)

PASS, Walter. Thematischer Katalog sämtlicher Werke Jacob Regnarts (ca. 1540 - 1599). (=Tabulae Musicae Austriacae 5), Wien, Köln, Graz: Hermann Böhlau in Kommission, 1969.

PFUDEL, Ernst. Die Musik-Handschriften der Königl. Ritter-Akademie zu Liegnitz. In Beilage zu den Monatsheften für Musikgeschichte, Robert Eitner (ed.), 1. Bd., Leipzig: Breitkopf et Haertel, 1886, S. 5-74.

POPIGINIS, Danuta - SZLAGOWSKA, Danut. Musicalia Gedanensis. Rekopisy muzyczne z XVI i XVII wieku w zbiorach Biblioteki Gdańskiej. Gdańsk: Polskiej Akademii Nauk, 1990.

REGNART, Jacob. Missae sacrae ad imitationem... Frankfurt: Nikolaus Stein, 1602. (RISM A/I/7 R $734 / 9)$

RAJTER, Adrian. Missa Quodlibetica Jacobi Regnarti - príspevok k rekonštrukcii diela [Missa Quodlibetica Jacobi Regnarti - ein Beitrag zur Rekonstruktion des Werkes]. (Dipl. Arbeit), Bratislava: Filozofická fakulta Univerzity Komenského, 1994.

STEUDE, Wolfram. Bartfelder Handschriften. In Die Musik in Geschichte und Gegenwart, Sachteil 1, Ludwig Finscher (hrsg. v.), Kassel-Stuttgart: Bärenreiter-Metzler, 1994, Sp. 1257.

Tabulatúrny zbornik Samuela Marckfelnera / Tabulaturbuch des Samuel Marckfelner (Výber / Auswahl). František Matúš (ed.), Bratislava: OPUS, 1981.

VEIT, Dietrich. Agend Büchlein... Nürnberg: Berg \& Newber, 1553.

WICK, Vojtech. Dóm svätej Alžbety v Košiciach [Dom der Heiligen Elisabeth in Košice]. Košice: Nákladom mesta Košíc, 1936. 
